\title{
The beginning of Edoardo Amaldi's interest in gravitation experiments and in gravitational wave detection
}

\author{
Adele La Rana* \\ TERA Foundation, Novara, Italy \\ \&3 \\ Sapienza University, Rome, Italy \\ *E-mail: Adele.LaRana@roma1.infn.it \\ Luisa Bonolis \\ Max Planck Institute for the History of Science, \\ Berlin, Germany \\ E-mail: l.bonolis@mpiwg-berlin.mpg.de
}

\begin{abstract}
Unedited documents and letters* allowed to establish that Edoardo Amaldi's first interests in experiments on gravitation date back to the late 1950s, about twelve years before the beginning of the research activity in gravitational wave (GW) detection in Rome (1970). Amaldi was connected to the main protagonists of the historical phenomenon that many historians call the Renaissance of General Relativity (GR), characterised by the new attitude of the scientific world towards Einstein's theory of gravitation, which had its start in the middle of the 1950s and which grew along the 1960s, with the birth of relativistic astrophysics ${ }^{\dagger}$. Since the second half of the 1960s, Amaldi's will of beginning an experimental activity for detecting gravitational radiation clearly emerges.
\end{abstract}

Keywords: Gravitation; general relativity; gravitational waves; Edoardo Amaldi; Bruno Touschek; Robert Dicke; Dmitri Ivanenko; Robert Wheeler; Joseph Weber; Livio Gratton; Remo Ruffini.

\section{Introduction: The known story}

In an internal note from the Institute of Physics of Rome published in $1975^{\mathrm{a}}$, Edoardo Amaldi recounts:

The idea of starting an experiment aiming to detect $G W$ in Rome was stimulated by the Course on Experimental Tests of Gravitational Theories held in summer 1961 at the Scuola Internazionale E. Fermi in Varenna, where the problem was discussed by J.Weber. The program remained rather vague for practical reasons until 1968, when W. Fairbank spent a few months in Rome at G. Careri's low temperature laboratory. When Fairbank mentioned

\footnotetext{
*The unedited documentation cited in the present paper comes mainly from: Edoardo Amaldi's Archives, Department of Physics G. Marconi - Sapienza University (Rome); Archives of the Rectorate of Sapienza University, Professors' personal folders; Archives of the National Research Council (CNR), via dei Taurini (Rome)

${ }^{\dagger}$ More details about the Renaissance of GR and its developments in Italy can be found in this volume in the paper by L. Bonolis, R. Lalli, A. La Rana, The Renaissance of General Relativity in Rome: Main Actors, Research Programs and Institutional Structures.

${ }^{a}$ E. Amaldi, G. Pizzella, The gravitational wave experiment in Rome: progress report, Nota Interna n. 645, Istituto di fisica G. Marconi, Università di Roma, November 10, 1975.
} 
his intention of starting the development of a low temperature gravitational antenna, Careri who was informed for long time of the interest of E. Amaldi in the subject, suggested a first direct contact. This was the beginning of the collaborations between the groups of Stanford (W. Fairbank), Louisiana State University (W. Hamilton) and the Istituto di Fisica of Rome. Already at this stage a number of conversations with $R$. Ruffini stimulated the beginning of these researches.

The research activity in GW detection in Rome started officially in September 1970. Guido Pizzella, Edoardo Amaldi's assistant, had just come back from the USA, where he had been working at the University of Iowa with James Van Allen, when he proposed to Amaldi to begin an experiment in this field.

The interest in GWs had been growing in the scientific community since the end of 1960s, stimulated by the works of Joseph Weber, the pioneer of GW detection and the inventor of GW resonant bar detectors. Between 1967 and 1969 Weber published few papers claiming with increasing confidence to have detected gravitational radiation, by the room temperature resonator set up in his laboratory at the University of Maryland ${ }^{\mathrm{b}}$. Furthermore the discovery of pulsars in 1968 provided an attractive candidate as GW source ${ }^{c}$.

The roman group was originally composed by: Edoardo Amaldi, Massimo Cerdonio, Renzo Marconero, Ivo Modena and Guido Pizzella. It was the first team in Italy working at GW detection and one of the first to be born in Europe. While W.D. Allen at the University of Reading and P.S. Aplin in Bristol were constructing room temperature Weber type detectors, the Rome-Stanford-Louisiana collaboration envisaged the construction of three cryogenic detectors, cooled to $0.003 \mathrm{~K}$, to be installed in the three locations and working in coincidence.

The story narrated by Amaldi and what comes after have been recounted in different occasions and articles by the protagonists. The present paper traces instead the unedited story of what happened before these events, highlighting the evolution of Amaldi's first approaches to gravitation experiments.

\footnotetext{
${ }^{\mathrm{b}}$ Weber reported the first possible gravitational wave signals in the paper Weber J. (1967), Gravitational Radiation, Phys. Rev. Lett. 18, 498-501, describing occasional mechanical excitations of the detector well above mean thermal-noise excitations. The paper was soon followed by: 1) Weber J. (1968), Gravitational-Wave-Detector Events, Phys. Rev. Lett. 20, 1307-1308; 2) J. Weber (1969), Evidence for Discovery of Gravitational Waves, Phys. Rev. Lett. 22, 1320-1324. Weber's papers turned on a lively scientific debate, stimulating the birth of GW detection groups in other parts of the world.

${ }^{\mathrm{c}}$ In August 1968, few months later the announcement of the discovery of pulsars, Joseph Weber published: Weber J., Gravitational Radiation from the Pulsars, Phys. Rev. Lett. 21, 395.
} 


\section{The unedited origins}

Amaldi was one of the main actors of the rebirth of Italian and European Physics after the Second World War. A true statesman of science, as Carlo Rubbia defined him $^{\mathrm{d}}$, he was the first Secretary General of CERN, during the delicate phase which brought to the definitive affirmation of the European Laboratory project and the final ratification of the member states (1952-1954). In the following years he gave a fundamental contribution also to the foundation of the European Space Research Organisation (ESRO), born in 1964, the first nucleus of the future European Space Agency.

Between 1957 and 1960 Amaldi was the President of the International Union of Pure and Applied Physics (IUPAP). During his presidency the International Committee on Gravitation and Relativity, chaired by André Lichnerowicz and MarieAntoinette Tonnelat, was constituted and entered the agenda of IUPAP. In a letter addressed to Dmitri Ivanenko, well-known relativist and member of the committee, Amaldi wrote $\mathrm{e}^{\mathrm{e}}$ I think this is a right moment for a wider development of the research activity on gravitation and general relativity.

By the end of 1950s, Amaldi was clearly aware of the new lymph that was flowing in the field of GR. The constitution of a specific GR community, the organization of periodical international conferences and the new theoretical and experimental investigations about gravity, which were taking place in the second half of the 1950s, marked the beginning of the so called Renaissance of $\mathrm{GR}^{\mathrm{fg}}$.

Connected to the major international physicists, Amaldi was attentively following the renewed interest of the scientific community in the experimental tests of GR, in particular Robert Dicke's program of precision measurements of the equivalence of inertial and gravitational mass. In February 1960 he had sent indeed a letter to Dicke, asking him preprints and internal notes about the results of his experiments. On September 3, after the Rochester Conference held in August, Amaldi and his young colleague Giuseppe, Fidecaro visited Dicke in Princeton and could see his experimental facility ${ }^{\mathrm{h}, \mathrm{i}}$.

\footnotetext{
${ }^{\mathrm{d}}$ Rubbia C. (1991), Edoardo Amaldi, scientific statesman, CERN Yellow Report 91/09, Geneva. ${ }^{\mathrm{e}} \mathrm{E}$. Amaldi to D. Ivanenko, 22th of August 1960.

${ }^{\mathrm{f}}$ Blum A., Lalli R., Renn J. (2015), The Reinvention of General Relativity: A Historiographical Framework for Assessing One Hundred Years of Curved Space-time, Isis Vol. 106, No. 3, pp. 598-620.

gEisenstaedt J. (1989), The low water mark of general relativity, 1925-1955, in Einstein and the history of general relativity (Einstein Studies, vol.1), ed. D. Howard and J. Stachel, Birkhuser, Boston, pp. 277292.

${ }^{\mathrm{h}} \mathrm{E}$. Amaldi to R. Dicke, 28th of July 1960. In the letter Amaldi wrote explicitly: I would be very glad if I could see your experiments on the equivalence of inertial and gravitational mass.

${ }^{\mathrm{i}}$ It was possible to identify the date of the visit to Dicke through Amaldis diaries. Amaldi used to keep very detailed diaries of his activities, so it was possible to find accurate notes and drawings about Dickes discussion. In a recent personal email to Ugo Amaldi, Edoardo's son, and to the authors of this paper, Fidecaro recounted: Dicke discussed his experiments with us for a couple of hours, in front of a big blackboard. Then he called somebody which I cannot remember and we went together to visit the laboratory.
} 
However, more than one year before Amaldi himself together with his colleague Mario Ageno had begun a few experiments concerning gravitation. On Saturday 31st of January 1959, Amaldi took note in his diary: in the afternoon he had spoken with Ageno about the possibility of looking for an effect of the gravitational field on the beta disintegration; the idea came from a discussion with Bruno Touschek, the father of the first electron-positron collider AdA, on the morning of Wednesday 28th of January. The question raised by Bruno Touschek was expressed by Amaldi and Ageno in a paper published in 1966 by the Accademia dei Lincei ${ }^{\mathrm{j}}$ : Is the decay constant of the various beta decay nuclei the same everywhere in the Universe, irrespective of the value of the local gravitational field?

Already one week later, the first experiment had been set up and accomplished; it was based on the equivalence principle, according to which a centrifugal field generated by a rotation is locally equivalent to a gravitational field produced by a proper distribution of masses. Amaldi and Ageno accelerated a sample of beta radioactive source inside a centrifuge, measuring its activity repeatedly as a function of time, before and after centrifugation.

They discovered only later that other researchers in the world were dealing with the same problem, as Chien-Shiung Wu ${ }^{\mathrm{k}}$. Amaldi's and Ageno's experiments, accomplished at the Istituto Superiore di Sanità in Rome, continued in the following years, but their results have never been published on international reviews ${ }^{\text {. }}$

Another important stimulus towards gravitation experiments came to Amaldi from Dmitri Ivanenko, who visited Italy during January and February of 1960. On January 26th, few days after Amaldi's discussion at CERN with Møller, Ivanenko gave a talk at the Intistute of Physics in Rome, entitled Remarks on transmutation of matter into gravitation ${ }^{\mathrm{m}}$. As shown by his diaries, Amaldi was present. He had come back from CERN on January 21 and would go back in Geneva on February 3 for the PS inauguration (5th of February). He wrote down some very interesting annotations about Ivanenko's talk. The Russian physicist had developed a quantum-gravity model predicting transmutations of ordinary particles in

\footnotetext{
${ }^{\mathrm{j}} \mathrm{M}$. Ageno, E. Amaldi, Experimental search for a possible change of the beta decay constant with centrifugal force, Atti della Accademia Nazionale dei Lincei, Anno CCCLXIII, 1966, Memorie. Classe di Scienze fisiche, matematiche e naturali, Serie VIII, Vol. VIII, Sez. 2, Fascicolo 1, Roma. ${ }^{\mathrm{k}}$ Wu, C. S. (1959), Parity Experiments in Beta Decays, Reviews of Modern Physics, 31, 783.

${ }^{1}$ Amaldi discussed the beta decay issue with physicists from different institutes, as we learn from his diaries and letters. On January 19 of 1960, few days before the inauguration of CERN's first accelerator, the Proton Synchrotron (PS), he talked in Geneva with Christian Møller about the half life of centrifugated pions (Amaldi wrote about this conversation in one of his diaries). In November 1964 he wrote to John Synge asking him whether to publish or not their results, but no reply was found in Amaldi's archives. He continued to think about the centrifuge experiments in the following years, as witnessed by the letters he wrote in 1973-74 to Møller and to Francis Perrin, sending them a copy of the 1966 paper published by the Accademia dei Lincei and asking their opinion.

${ }^{\mathrm{m}} \mathrm{E}$. Amaldi, Centro di studio per la fisica nucleare e delle particelle elementari. Attività svolta durante l'anno 1959-1960. Estratto da La Ricerca Scientifica, 1962, Consiglio Nazionale delle Ricerche Roma.
} 
a graviton, by analogy with creation and annihilation of electron-positron pairs in an electromagnetic quantum. Amaldi followed attentively his reasoning and noted in Italian: It is necessary to study gravitational waves, because it is not sure they exist.

Finally, as recounted in the internal note at the beginning of this paper, Amaldi participated in the Varenna School on the Experimental Tests of Gravitational Theories (19th June - 1st July), directed by Møller. Among the lecturers were Bruno Bertotti (Laboratorio Gas Ionizzati, Frascati), Herman Bondi (King's College, London), Robert Dicke (Princeton University) and Joseph Weber (University of Maryland). Amaldi was present in the days 27th, 28th and 29th of June and, among his notebooks, there is one specifically dedicated to this Varenna School. Strangely, it does not contain any reference to Weber's talk about GW detection ${ }^{\mathrm{n}}$. Furthermore, the exchange of letters between Amaldi and Weber is very poor, also taking into account the importance Amaldi attributed to his 1961 encounter with him ${ }^{\circ}$. During the 1960s there was only one short letter written by Weber to Amaldi ${ }^{\mathrm{p}}$ : Weber invited Amaldi to visit his laboratories at the University of Maryland; as learned from the documents explored so far, this did not happen until March 1980.

Besides Amaldi's quest for fundamental physics, the new astronomical discoveries, such as quasars (1963) and pulsars (1967), triggered his interest in gravitational radiation. Together with multiwave astronomy, cosmic rays, particles from the solar wind, and neutrinos, gravitational-wave astronomy might open another window on the universe. And indeed, during the 1960s Amaldi promoted the opening up of new lines of research in Rome, like space science, plasma physics and astrophysics, calling back at the Institute of Physics scientists as Livio Gratton, for whom the first chair of astrophysics in Rome was established in 1962; encouraging the training abroad of young researchers as Guido Pizzella; stimulating synergies among different expertises and actively contributing to the creation of dedicated laboratories as the Ionized Gas Laboratory in Frascati ${ }^{\mathrm{q}}$.

As a young student, Remo Ruffini had a privileged role in those early years. In 1967 Amaldi strongly supported his application for an ESRO fellowship, which would allow him spending two years in USA. In his letter of July 7 to Reimar Lüst (who was about to become the Vice President of ESRO, in 1968), Amaldi explained that Ruffini would work both experimentally (Dicke) and theoretically (Wheeler) at Princeton and would spend some time at the Maryland University (Weber), training on gravitation. Amaldi's explicit aim was to set up an experimental group working

\footnotetext{
"Instead, accurate annotations about Bruno Bertotti's lectures are found, discussing the topic The theory of measurement in general relativity. See: Bertotti B. (1962), The theory of measurement in general relativity, in Rendiconti della Scuola Internazionale di Fisica Enrico Fermi. XX Corso. Verifiche delle teorie gravitazionali, ed. C. Mller, Academic Press, New York and London.

${ }^{\circ}$ E. Amaldi, G. Pizzella, The gravitational wave experiment in Rome: progress report, Nota Interna n. 645, Istituto di fisica G. Marconi, Università di Roma, November 10, 1975.

p J. Weber to E. Amaldi, 15th of September 1967.

qFor further details see the paper by L. Bonolis, R. Lalli, A. La Rana, The Renaissance of General Relativity in Rome: Main Actors, Research Programs and Institutional Structures in this volume.
} 
in this field at the return of Ruffini: Just to mention one possible line of research, we are considering the possibility of establishing coincidences between detectors of gravitational waves placed at large distances (one in USA and one in Italy).

In a previous letter dated April 14 of 1967 to the National Research Council (CNR), concerning a six months fellowship of Ruffini in the USA, Amaldi wrote explicitly: The aim of creating an experimental group on gravitation has a great interest for this Institute and in particular for me personally ${ }^{\mathrm{r}}$. On February 9 of the following year, Ruffini wrote a letter to Amaldi attaching an activity report he had prepared for ESRO, in order to support the renewal of his fellowship for a second year at Princeton University. Here is a paragraph particularly significant for our story:

Researches on gravitation are not by any means new at Rome University. Quite apart from earlier work on gravitation theory of Tullio Levi Civita and Enrico Fermi, significant investigation have been carried out in this field in the past few years (measurement of muon and beta decay in non-inertial frames; theory of superdense stars; theory of gravitational radiation).

A further historiographic re-enactment is needed to make a clear picture about these first studies on gravitational radiation in Rome in the 1960s and the early 1970s.

Remo Ruffini had an important role in stimulating the beginning of the research activity in GW detection in Rome and the start-up of the contacts between Amaldi's group in Rome, William Fairbank's group in Stanford and William Hamilton's team in Lousiana State University. However, the man who actually materialized Amaldi's expectations and founded the experimental group was Guido Pizzella, who undertook with courage, enthusiasm and perseverance the leadership of the new scientific adventure.

\footnotetext{
${ }^{\mathrm{r}}$ Today the Edoardo Amaldi Conference on $G W s$ is one of the most important international gathering in the field of GWs. The first one was organized in Frascati in 1994 by Eugenio Coccia; the second conference, in 1997, took place in Geneva at CERN, with a massive participation of the GW community. During the latter conference, the GW International Committee (GWIC) was born, under the chairmanship of Barry Barish, and it was decided that the next conference would be held in Caltech (1999). Since then, the Edoardo Amaldi Conference on GWs takes place every two years in a different country of the world and concerns all the aspects of GW research, from theoretical models of astrophysical sources to detectors and signal processing. This brief note is based on the paper: Coccia E. (2009), Edoardo Amaldi and the birth of the gravitational wave research in Italy.
} 